LETTERS

\section{The role of tricyclic antidepressants and tramadol in palliative care}

We read with interest the review article on alternative opioids to morphine in palliative care. ${ }^{1}$ The author has mentioned in detail various factors-biomedical, genetic, and psychological-which influence the effect of opioids. Though most of the aspects are well covered, the role of depression has not been discussed, and this has a tremendous impact on the manifestation and management of pain. $^{2}$ Initial control of depression greatly facilitates pain management. Depression must be treated aggressively (for example with antidepressants and psychotherapy sessions) or pain management will remain elusive. It has also been shown that in patients who are taking opioid drugs, the bioavailability of opioids is increased with antidepressants; the tricyclic drugs are membrane stabilising, which may account for the early onset of action in patients with chronic pain.

The author mentioned the advantages of transdermal administration of fentanyl. She noted that it is highly acceptable to patients and the patches can be applied by patients or relatives themselves. We would like to stres that $25 \%$ to $50 \%$ of patients above the age of 65 suffer from major pain problems. Age related changes in skin integrity, subcutaneous fat, and water content can affect patien response to transdermal products. In fact, fentanyl patches have been associated with death in opioid-naive older adults in doses as low as $50 \mathrm{\mu g} /$ hour. $^{2}$ Also, serum fentanyl concentrations may increase by one third in patients with a body temperature of $40^{\circ} \mathrm{C}$ or more. It has been suggested that fentanyl should not be given to children younger than 12 years of age or to patients younger than 18 years of age who weigh less than $50 \mathrm{~kg}$. Additionally, fentanyl has a long duration of action (up to 72 hours) and therefore the side effects and adverse reactions are not easily reversed. In view of this, we believe that transdermal fentanyl should not be used liberally.

It was also stated by the author that tramadol is less potent than morphine and less effective for managing severe pain. However tramadol has been used extensively and evaluated over the last 20 years. It has proved as effective as the strong opioids in acute and chronic pain settings. In particular, tramadol administration results in little respiratory depression in comparison with equianalgesic doses of opioids, such as morphine or pethidine. Tramadol has a long record of efficacy and safety, and although it should be avoided or used with caution in epileptic patients, it is now the fourth most commonly prescribed analgesic worldwide. ${ }^{5}$ It is certainly useful in the treatment of chronic, non malignant, and malignant pain syndromes Another considerable advantage of tramadol is its very low abuse potential. Consequently, it is not deemed a controlled (scheduled) drug.

In view of the above, we believe that tramadol has an important role as an alternative opioid to morphine in palliative care.
M Thulasimani

Department of Medicine Community Health Center, Mannadipet, Pondicherry 605 501, India Prakram@md4 vsnl.net.in

S Ramaswamy

Department of Clinical Pharmacology, Jawaharla Institute of Postgraduate Medical Education and Research, Pondicherry 605 006, India

\section{References}

1 Barnett M. Alternative opioids to morphine in palliative care: a review of current practice and evidence. Postgrad Med J

2001;77:371-8.

2 Gloth III FM. Geriatric pain. Factors that limit pain relief and increase complications. Geriatrics 2000;55:46-52

3 Hardy PA. Analgesics. In: Hardy PA, ed Chronic pain management: the essentials. London: Greenwich Medical Media, 1977: 31-6.

4 Salemo E. Analgesics. In: Salemo E, ed. Pharmacology for health professionals. USA: Mosby, 1999: 124-53.

5 Bamigbade JA, Langford RM. Tramadol hydrochloride: an overview of current use. Hospital Medicine 1998;59:373-6.

\section{Author's reply}

First, I would like to address the apparent omission of a discussion of depression. I am entirely in agreement with the respondents' comments that depression has a major role in the manifestation and management of pain. However, in this review I was considering the differential factors influencing choice of opioid, not the assessment of pain per se. I pointed out at the outset that cancer pain was multifactorial, and that a thorough assessment was a prerequisite to successful management, but that the review was of strong opioids, and was beginning from the point at which these were considered appropriate treatment. I did include the effect of antidepressants in enhancing bioavailability of opioids in the text and in table 5, but in the context of drug interactions.

With regard to transdermal fentanyl: the authors appear to imply that in beginning with a discussion of its advantages, I was advocating liberal use of this drug and route. I went on to describe all its disadvantages, including its interpatient variability and long duration of action causing potential problems of overdose. I was attempting to provide a balanced overview of a drug that has proved very popular with patients and health professionals while cautioning on its overuse.

On the question of tramadol, I do not dispute that it is an effective analgesic and has a broad spectrum of clinical use. However, both from personal practice and my review of literature pertinent to palliative care, I concluded that its efficacy in the management of progressive severe cancer pain is less conclusively demonstrated, and thus questioned its role in palliative care. Respiratory depression is rarely an issue in palliative care, nor opioid abuse, although I accept that low abuse potential may facilitate adequate analgesic prescribing in chronic non-malignant pain.

\section{Watermelon poisoning}

Food borne diseases are a result of ingestion of foods contaminated by either infectious or toxic agents. These diseases are sometimes inaccurately referred to as "food poisoning" and they represent one of the most widespread and overwhelming public health probems of the modern world. Infants, children, the elderly, and the immunocompromised are more commonly affected. ${ }^{1}$ Infection of six members of a family is described here.

The head of a family (61 years), his wife (59 years), their son (38 years), daughter in law (35 years), and two male grandchildren (14 and 11 years respectively) were admitted to hospital with gastroenteritis. About four hours earlier they had consumed pieces of freshly cut watermelon. During the past seven days they had consumed home cooked food and clear water from the domestic supply. The head of the family, who had received the lion's share of the fruit, was affected the most and was in a state of shock and acute renal failure. It took three days for his urinary output and renal parameters to improve. He was treated with intravenous fluids, ciprofloxacin, metronidazole, and other conservative measures for five days. Other members of the family had an uneventful stay in the hospital and were discharged on the second day after admission. The daughter in law, who had received the smallest share of the fruit, was affected the least and had just two or three loose stools in hospital. Haematology, urinalysis, and chest radiography of all the family members were normal. Blood biochemistry of the head of the family suggested uraemia and acidosis. Stool cultures of all members of the family grew an enteroinvasive variety of Escherichia coli, which was non-motile with non-lactose ferments.

We asked the fruit seller about the purchase of watermelon and this revealed the fact that watermelons can be made more colourful and sweet without cutting them open. Instead a long needle, into the core, can inject sweetener and colouring agents, three to four hours before sale. The nature of the injected agents was not revealed by the fruit seller for obvious reasons. Culture from the solution that had been injected (which had been prepared and stored in an earthenware bowl), also grew multiple colonies of the enteroinvasive variety of $E$ coli, which were biochemically lactose positive, non-motile, with non-lactose ferments.

Diarrhoeal diseases have been commonly attributed to a pathogen contaminated water supply, but it is now recognised that food also plays an equally important part in $70 \%$ of such illnesses. Besides the usual foods, contamination has been reported in other foods such as raw fish, shellfish, bivalve molluscs (oysters, cockles, mussels), raw shrimp, pork mixed d'oeuvre, crabs, prawns, rock lobster, cooked squid, turkey, street foods, eggs, egg salad, cold asparagus, aquatic plants, bottle feeds (for infants), ice creams, chocolates, candies, etc. The chief contaminants are bacteria ( $E$ coli, shigella, salmonella, Vibrio cholera 01, Campylobacter jejuni, brucella, Bacillus cereus, Staphylococcus aureus, Clostridium perfringens, and Clostridium botulinum) helminthes (Trichinella spiralis, Taenia saginata Taenia solium, clonorchis, Fasciola opisthorchis, Paragonimus spp), protozoa (Entamoeba hystolitica, Giardia lamblia, Cryptosporidium spp), and enteric viruses (rotavirus hepatitis A\&E virus) etc. ${ }^{1}$ 
Infections due to pathogenic strains of $E$ coli are probably the commonest cause of diarrhoea in developing countries. The contamination of food with micro-organisms is caused by:

- Use of contaminated equipment.

- Infected food handlers.

- Use of raw and contaminated ingredients.

- Cross contamination.

- Addition of toxic chemicals or use of foods containing natural toxicants like mushrooms etc. ${ }^{1}$

Gastroenteritis by Salmonella javiana contamination of watermelon has been described in 26 cases in the USA. ${ }^{2}$ Contamination of fruit by such a novel method as described here may prove to be a major public health hazard, and hence is noteworthy.

T K Pande, A H Khan, R Pipersania, S K Sethi, Y Rath

Department of Internal Medicine, J L N Hospital and Research Center, Bhilai 490 009, India; pandetk@mantraonline.com

\section{References}

1 World Health Organization. Food borne disease: a global health and economic problem. Food borne disease: a focus for health education. Geneva, Switzerland: WHO, 2000: 1-34.

2 Blostein J. An outbreak of Salmonella javiana associated with consumption of watermelon. Journal of Environmental Health 1993;56:29-31.

\section{BOOK REVIEWS}

The reviewers have been asked to rate these books in terms of four items: readability, how up to date they are, accuracy and reliability, and value for money, using simple four point scales. From their opinions we have derived an overall "star" rating: * $=$ poor, ${ }^{* *}=$ reasonable, ${ }^{* * *}=$ good, ${ }^{* * * *}=$ excellent

\section{A Practical Guide for Medical Teachers.}

Edited by John A Dent and Ronald M Harden. (Pp 453; £34.95.) Churchill Livingstone, 2001. ISBN 0-443-06273-0. *** *

This book tells you all you wanted to know and a good deal more that you now need to know about the revolution that has been taking place in undergraduate medical education in the past decade or more. The editors' stated intention is to provide jargon-free understanding of contemporary educational principles and provide practical advice on dealing with all aspects of teaching. They succeed brilliantly well.

The book is divided into seven sections (totalling 39 chapters in all) starting with aspects of the curriculum, learning situations such as lectures, small groups, wards, primary care etc, educational strategies (independent, problem based, integrated and multiprofessional), aids such as computers, audiovideo, and guides, themes (basic sciences, communication skills, ethics, informatics etc), means of assessment, and aspects of students and staff such as selection, support staff development, and course monitoring.
There is evidence of tight editing in terms of chapter style and layout and excellent cross referencing between the chapters. The book is enlivened by quotations in wide easy-on-theeye margins. These also host simple line drawings of such important practical aspects of teaching as the ideal distribution of teacher and taught round a bed. The advice to ensure students do not go off on electives to countries with military or civil unrest is not profound but well timed. Each chapter provides suggestions for further reading.

The principles and examples are, as the editors suggest, relevant to postgraduate medical education and other healthcare professionals. But for those involved in the teaching of medical students, this book from Dundee, a teaching centre of excellence, is warmly recommended for those who want to improve their understanding and performance and hence enjoyment of this Hippocratic responsibility.

\section{Fellowship of Postgraduate Medicine, \\ Making Sense of Statistics and Healthcare.}

B I Hoffbrand London, UK

By Anna Hart. (Pp 162; £19.95.) Radcliffe Medical Press, 2001. ISBN 1-85775-472. $7 * \star \star *$

This book describes the essentials of statistics and data presentation. The first chapters deal with the different types of study designs, the various forms of data and the characteristics of pictorial forms of presenting data. Subsequent chapters deal with distributions, means and medians, and measures of variability. The author describes confidence intervals, p values, statistical power, and type I and II errors. The final sections are on confounders, interactions, measures of correlation, hierarchy of evidence, causality, and how to assess papers. The author describes the terms used in evidence based medicine such as absolute risks and the number needed to treat. A glossary of terms used is contained in the appendices.

The book will be of invaluable use for those just starting a research career or in its early stages. It is comprehensive and clearly written with many illustrative examples. The text covers well all the areas that are of interest to those commencing their own research projects. The pitfalls of different tests are well described and the book is competitively priced.

A R Hart

Consultant Gastroenterologist West Norwich Hospital, UK

\section{Fast Facts-Sexually Transmitted Infections.}

By Ann Edwards, Jackie Sherrard, and Jonathan Zenilman. (Pp 104; £12.00.) Health Press, 2001. ISBN 1-899541-04-7.****

In a month when all GPs in my area have been notified of an epidemic of syphilis in London, the publication of this book could not be more timely.

There has long been a need for a concise, affordable, accessible, and accurate textbook on sexually transmitted infections (STIs) for non-specialists in genitourinary medicine and this volume fills the gap perfectly. The clear, sharp, and relevant photographs and illustrations are an added bonus and the tables such as those of causes of vaginal discharge and genital ulceration are exemplary in their clarity and comprehensiveness. Relevant aspects of dermatology and even psychiatry are briefly considered where appropriate.

Three opening chapters on aetiology of STIs, taking a sexual history and counselling approaches, are followed by chapters dealing with STIs causes by bacterial, viral, and other organisms in turn. For once a correct balance (in terms of their respective incidence in the UK) is shown between coverage of HIV and non-HIV STIs. Other chapters are problem oriented and consider such commonly presenting conditions as urethritis and pelvic inflammatory disease. Comprehensive treatment guidelines for both the UK and USA conclude the book.

As STIs continue to rise in the UK, this text will prove an indispensable ready reference guide for paediatricians, physicians, GPs, gynaecologists, dermatologists, and surgeons. I will add my copy to the other three essential reference books on my surgery desk.

T G Stammers General Practitioner Merton Park, London, UK

\section{MCQs in Paediatrics for MRCPCH Part I.}

By Nagi G Barakat. (Pp 212; £14.95.) Royal Society of Medicine Press, 2001. ISBN 1-85315-491-1. **

This paperback book in fact has 463 questions including a 60 question examination. The format is of MCQs as used in the part I of the MRCPCH examination and the question material does cover the full syllabus for this. The answers provide some explanations for the questions but not all. The evidence for the answers isn't related to specific text. However, there are a number of recommended texts and references listed, but at least one of these texts, that for neonatology, does not show the most recent edition edited by Roberton and Rennie.

As with all MCQ questions, some of the answers are arguable but there are certain answers given that are clearly completely wrong, which will further mislead the examination candidate.

Although the material covers the syllabus, the questions that have been prepared do not reflect the quality of the questions expected in the membership examination.

Given the thirst for examination questions among membership candidates, however, it is likely that some candidates will find the book useful in their preparation.

A C Elias-Jones Consultant Paediatrician, Leicester General Hospital, and Examination Committee,

Royal College of Paediatrics and Child Health, UK

\section{Imaging Picture Tests for the MRCPCH.}

By A P Winrow. (Pp 205; £ 19.95.) Churchill Livingstone, 2000. ISBN 0-443-06445-

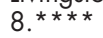

This is a useful paediatric teaching book for candidates studying for the MRCPCH final examination. It may also be useful for 
candidates for the Diploma for Child Health. It contains a selection of 100 varied paediatric and neonatal cases of the types commonly seen in the examination. There is an easily accessible question and answer format on the following page with useful comments relevant to the condition being demonstrated There are also useful examination tips applied to each case

The author admits that some of the images were obtained from copy film and as a result some of the images are perhaps not as clear a they might have been. However, the examination candidate with the help of the answers and comments should be able to follow the demonstrated abnormalities.

The examination candidates will find this a useful additional text to assist their learning. It could also provide useful CPD for self assessment by already qualified paediatricians in addition.

A C Elias-Jones

Consultant Paediatrician Leicester General Hospital,

and Examination Committee,

Royal College of Paediatrics

and Child Health, UK

\section{DIARY}

\section{Fellowship of Postgraduate Medicine \& CHIME, Royal Free \& University College Medical School}

\section{Clinical Governance, Thursday, 21 February 2002}

A one day conference for doctors wishing to become more involved in clinical governance at the Commonwealth Conference Centre, Kensington High Street, London.

\section{PACES (MRCP) Part II}

Two two day courses designed as final preparation for the PACES examination to be held at the Whittington Hospital, London.

Communication Skills and Ethics: 7 and 8 February 2002. Cost: $£ 500$.

Clinical: 12 and 13 February 2002. Cost: $£ 500$. For details contact Kate Stephens, CHIME, 4th Floor Holborn Union Building, Archway Campus, Highgate Hill, London N19 3UA (tel: 020-7288-3134,email: k.stephens@chime. ucl.ac.uk)

\section{Thackray Museum}

25 April 2002-History of herbals, a talk by Bruce Madge of the British Library.

11 May 2002-Use and abuse: a history of opium, University of Leeds Medical History Day School.

For further information contact the Thackray Museum, Beckett Street, Leeds LS9 7LN (tel: 0113244 4343, fax: 01132470219 , email info@thackraymuseum.org, web site: thack raymuseum.org).

\section{CORRECTION}

\section{Hypokalaemia and hyperkalaemia}

An error occurred in the above paper, published in the December issue of the journal (2001;77:759-64). The name of the first author was spelt incorrectly; the correct spelling is A Rastegar. 